\title{
EVALUACIÓN DE MANIPULADORES ROBÓTICOS MEDIANTE MÉTRICAS DE CALIDAD DEL AGARRE
}

\author{
Carlos Rubert \\ Universitat Jaume I, carlos.ruibert@uji.es \\ Antonio Morales \\ Universitat Jaume I, antonio.morales@uji.es
}

\section{Resumen}

Durante los últimos años, se han realizado grandes avances en el desarrollo de manipuladores robóticos. Distintos métodos han sido utilizados para diseñarlos, permitiéndolos dividir en dos grupos, pinzas robóticas y manos artificiales antropomorfas.

En este artículo se utilizan un conjunto de métricas de calidad, ampliamente utilizadas en robótica, para analizar agarres, con el objetivo no sólo de comparar estos dos conceptos de diseño, sino también las limitaciones de las métricas a la hora de evaluarlos. Distintos manipuladores de ambos estilos de diseño y varios objetos son utilizados en un entorno de simulación donde las capacidades para coger son analizadas. Los análisis estadísticos sobre estos resultados permitirán estableces la dependencia de las métricas en el tipo de manipulador, pero también realizar evaluaciones comparativas en ambos métodos de diseño, proporcionando una evaluación que de como resultado un mejor rendimiento para realizar agarres.

Palabras clave: Manipulación robótica, métricas de calidad, evaluación.

\section{INTRODUCCIÓN}

El diseño y construcción de manipuladores artificiales hábiles es uno de los campos más activos en robótica. A lo largo de los años muchos diseño se han propuesto, con distintas tasas de éxito(figura 1). Analizando dichos diseños en conjunto se pueden observar dos tendencias de diseño distintas.

Por un lado está la construcción de pinzas robóticas que buscan un equilibrio entre la funcionalidad del diseño y su nivel de simplicidad y bajo coste. Esto ha llevado a dispositivos compuestos de hasta cuatro dedos, donde aspectos como la robustez, mecanismos subactuados, adaptabilidad y delicadeza han sido los mas relevantes. Este tipo de pinzas están enfocadas para ser utilizadas por robots en una variedad de tareas de manipulación y, su objetivo es proporcionar robots con dispositivos manipuladores competentes.
Figura 1: Modelos de manos robóticas. (a-d) Manos antropomorfas, (e-f) Pinzas robóticas. (a) Shadow

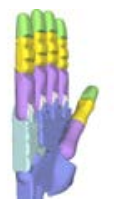

(e) PR2

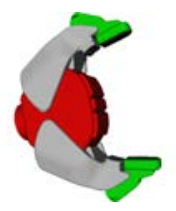

(b) Tact

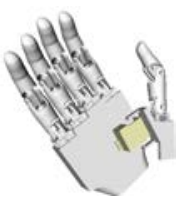

(f) Barrett

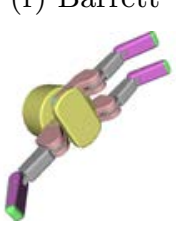

(c) Flexy

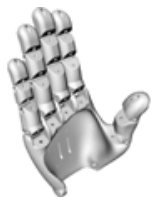

(g) Schunk

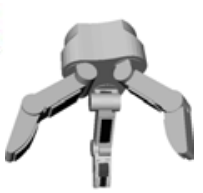

(d) Mich

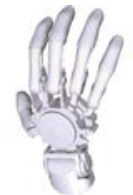

(h) Model T

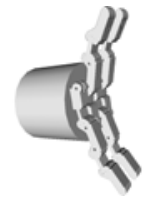

Por otro lato, la alternativa en el diseño ha sido la construcción de manos antropomorfas artificiales, cuya principal característica es su morfología y su parecido funcional con el de la mano humana. Estos dispositivos de 5 dedos se han caracterizado a menudo por su complejidad mecánica y la de su sistema de control. Se han utilizado tanto en robots humanoides como en entornos de investigación robótica, sin embargo, la robótica no ha sido su único campo de aplicación. Algunas manos antropomorfas artificiales se han diseñado también como dispositivos protésicos.

Durante los últimos años, la aparición de tecnologías de prototipado rápido y barato como las impresoras 3D y sistemas electrónicos como Arduino, han propiciado una avalancha de modelos de manos artificiales que pueden ser compartidas, obtenidas, construidas y controladas a precios asequibles. Esto es algo muy positivo, ya que permite la participación de muchos más agentes y, consecuentemente, incrementa la posibilidad de que nuevas ideas surjan.

Dado el auge de distintos diseños de manipuladores robóticos, surje una necesidad de evaluar y comparar las capacidades funcionales de cada mano. Esto se puede realizar llevando a cabo una serie de test experimentales en prototipos de manos, sin embargo, requiere de la preparación de un entorno experimental complejo y la fabricación de 
los prototipos de cada mano que debe ser evaluada.

Para superar estas dificultades, nosotros proponemos como metodología de evaluación testeo exhaustivo de modelos virtuales de manos artificiales en un entorno de simulación, donde las manos son configuradas para agarrar de distintas formas una gran variedad de objetos. Para cada configuración de agarre, se calculan diez métricas de calidad y el conjunto total de datos obtenidos se analiza estadísticamente, con el objetivo de establecer un rendimiento global de cada mano para cada una de las métricas seleccionadas.

Este artículo presenta un estudio comparativo de ocho modelos de manos, cuatro antropomorfos y cuatro pinzas robóticas, siguiendo la metodología anterior descrita. El objetivo de este estudio es establecer donde están las principales diferencias funcionales entre manos antropomorfas y pinzas robóticas.

\section{MÉTRICAS DE CALIDAD}

En anteriores trabajos (Leon et al.[1, 2]), se han revisado, seleccionado y adaptado un conjunto de 10 métricas de agarre comunes para estudiar distintos aspectos del agarre, y también el rendimiento de distintas manos (Rubert et al.[3]). Estos estudios consistieron en simular exhaustivamente los agarres de diversas manos robóticas sobre un conjunto de más de cien objetos. Para cada agarre obtenido, los valores de las métricas de calidad fueron calculados y el posterior análisis estadístico permitió determinar los rangos, correlaciones y sensibilidad de cada métrica. Los resultados permitieron establecer límites prácticos para el uso de métricas y sus relaciones.

La tabla 1 presenta un breve resumen de estas métricas con la notación definida en la Tabla 2. Estas métricas seleccionadas están clasificadas en 4 categorías dependiendo en sus principios subyacentes. las restricciones de la mano y el objeto son consideradas en la evaluación a través de los puntos de contacto, utilizando tanto la Matriz de Agarre G, como la localización de los puntos de contacto, o las fuerzas que deben ser aplicadas para resistir fuerzas externas. Adicionalmente, las restricciones de la mano se tienen también en cuenta a través de la posición de las articulaciones de los dedos o la medida de la capacidad para mover el objeto en cualquier dirección con la misma ganancia. Para cada métrica un factor de normalización es añadido para restringir el valor de la métrica a un intervalo entre 0 a 1 , siendo 0 el peor valor y 1 el mejor caso.

\section{METODOLOGÍA}

\subsection{MANOS ARTIFICIALES}

Para realizar este estudio, hemos seleccionado 8 modelos de manos artificiales: 4 pinzas y 4 manos antropomorfas (fig. 1). Las pinzas robóticas son PR2 Hand, Barrett Hand, Schunk Hand y Model T. La PR2 Hand[15] es una pinza de dos dedos, con 4 GdL (2 actuados y 2 subactuados). Las pinzas de Barrett[16] y Schunk[17] tienen 3 dedos con 7 GdL, 6 para el cierre y el grado de libertad extra para la abducción de los dedos. Por último, la Model T[18] es una pina robótica de 4 dedos actuados mediante tendones. La pinza puede ser construida utilizando tecnología de impresión 3D y las articulaciones y puntas de los dedos se construyen mediante materiales flexibles.

Para las manos antropomorfas hemos seleccionado las manos de Michelangelo, Shadow, Tact y FlexyHand. Las manos Tacthand[19] y Flexyhand[20] son modelos de código abierto disponibles para impresión 3D. La mano de Michelangelo[21] es una mano protésica ampliamente conocida, desarrollada por OttoBock y se encuentra comercialmente disponible. La ShadowHand [22] es una mano robótica también muy conocida desarrollada por Shadow.

La mano de Michelangelo es una mano protésica con 2 GdL y todos sus dedos emparejados a un mismo actuador, la abducción del pulgar está limitada a seis posiciones predefinidas. La TactHand es una mano protésica con $6 \mathrm{GdL}$, uno por dedo y uno extra para la abducción del pulgar, esta mano tiene dos articulaciones para cada dedo que están conectadas mediante un mecanismo de barras que las enlaza. La FlexyHand tiene tan solo $5 \mathrm{GdL}$, uno por dedo, esta mano carece de movilidad en la abducción del pulgar, sin embargo, los dedos restantes están operados por tendones, lo que hace que se acople con facilidad a la superficie de todo tipo de objetos.

La tabla 3 resume las especificaciones para el conjunto completo de modelos de manos.

Estos dos conjunto de manos son claramente diferenciables por sus características físicas. Las manos antropomorfas tienen 5 dedos, todos ellos de diferentes tamaños, pero al menos cuatro están alineados y el restante (pulgar) se encuentra opuesto a los otros. Esto provoca que los agarres dependan en gran mediada en la posición del pulgar, estando las fuerzas de contacto en el agarre centradas junto a los dedos pulgar, índice y corazón. 
Cuadro 1: Resumen de las métricas de calidad seleccionadas

\begin{tabular}{|c|c|c|c|c|c|}
\hline Nombre & & Formula & Normalización & Min & Max \\
\hline \multicolumn{6}{|c|}{ Group A: Algebraic properties of $G$} \\
\hline$Q_{A 1}$ & Smallest singular value of $\mathrm{G}[4]$ & $\sigma_{\min }(G)$ & - & 0 & - \\
\hline$Q_{A 2}$ & Volume of $G$ in the wrench space[4] & $\prod_{i=1}^{r} \sigma_{i}$ & - & 0 & - \\
\hline$Q_{A 3}$ & Grasp Isotropy Index[5] & $\sigma_{\min }(G) / \sigma_{\max }(G)$ & $Q_{A 3}$ & 0 & 1 \\
\hline \multicolumn{6}{|c|}{ Group B: Distribution of contact points } \\
\hline$Q_{B 1}$ & $\begin{array}{l}\text { Distance between the centroid of the contact } \\
\text { polygon and the object's center of mass }[6,7]\end{array}$ & distance $\left(p, p_{c}\right)$ & $1-Q_{B 1} /$ distance $_{\max }$ & 0 & 1 \\
\hline$Q_{B 2}$ & Area of the grasp polygon $[8]$ & $\operatorname{Area}\left(\operatorname{Polygon}\left(p 1, p 2, p 3, p 4_{P}, p 5_{P}\right)\right)$ & $Q_{B 2} / \operatorname{Area}_{\max }$ & 0 & 1 \\
\hline$Q_{B 3}$ & Shape of the grasp polygon[5] & $\frac{1}{\theta_{\max }} \sum_{i=1}^{n_{f}}\left|\theta_{i}-\bar{\theta}\right|$ & $1-Q_{B 3}$ & 0 & 1 \\
\hline \multicolumn{6}{|c|}{ Group C: Magnitude of Forces } \\
\hline$Q_{C 1}$ & $\begin{array}{l}\text { Smallest maximum wrench to be resisted }[9 \text {, } \\
\text { 10] }\end{array}$ & $\min _{w \in C W}\|w\|$ & $Q_{C 1} / \sqrt{2}$ & 0 & 1 \\
\hline$Q_{C 2}$ & Volume of the convex hull[11] & $\operatorname{Volume}(C W)$ & $Q_{C 2} /$ Volume $_{\max }$ & 0 & 1 \\
\hline \multicolumn{6}{|c|}{ Group D: Configuration of the manipulator } \\
\hline$Q_{D 1}$ & Posture of hand finger joints[12] & $1 / n_{q} \sum_{i=1}^{n_{q}}\left(\left(y_{i}-a_{i}\right) /\left(a_{i}-y_{i M}\right)\right)^{2}$ & $1-Q_{D 1}$ & 0 & 1 \\
\hline$Q_{D 2}$ & Inverse of the condition number of $\mathbf{G}_{\mathbf{J}}[13,14]$ & $\sigma_{\min }\left(G_{J}\right) / \sigma_{\max }\left(G_{J}\right)$ & $Q_{D 2}$ & 0 & 1 \\
\hline
\end{tabular}

\section{Cuadro 2: Notación}

\begin{tabular}{|c|c|}
\hline$G$ & Matriz de Agarre \\
\hline$r$ & Rango de $\mathrm{G}$ \\
\hline$\sigma_{\min }$ & Valor Singular Mínimo \\
\hline$\sigma_{i}$ & Valores Singulares no-zero \\
\hline$\sigma_{\max }$ & Valor Singular Máximo \\
\hline$p$ & Centroide del polígono de contacto \\
\hline$p_{c}$ & Centro de masa del objeto \\
\hline pi & Vértice del polígono de agarre \\
\hline$p i_{p}$ & $\begin{array}{l}\text { Proyección del vértice del polígono de aga- } \\
\text { rre sobre un plano }\end{array}$ \\
\hline$n_{f}$ & Número de dedos \\
\hline$\theta_{\max }$ & $\begin{array}{l}\text { Suma de las diferencias entre los ángulos in- } \\
\text { ternos cuando el polígono tiene la forma del } \\
\text { número de condición y aquellos del polígono } \\
\text { regular }\end{array}$ \\
\hline$\theta_{i}$ & $\begin{array}{l}\text { Ángulo interno en el vértice }{ }_{i} \text { del polígono } \\
\text { de agarre }\end{array}$ \\
\hline $\bar{\theta}$ & $\begin{array}{l}\text { Ángulo medio de todos los ángulos internos } \\
\text { del polígono de agarre }\end{array}$ \\
\hline$C W$ & $\begin{array}{l}\text { Envolvente convexa de las fuerzas primiti- } \\
\text { vas }\end{array}$ \\
\hline $\begin{array}{l}w \in C W \\
\|w\|\end{array}$ & $\begin{array}{l}\text { Fuerzas generalizadas actuando sobre CW } \\
\text { magnitud de una fuerza }\end{array}$ \\
\hline$n_{q}$ & Número de articulaciones de la mano \\
\hline$a_{i}$ & $\begin{array}{l}\text { Posición media del rango de una articula- } \\
\text { ción }\end{array}$ \\
\hline$y_{i}$ & Ángulo de la articulación $i$ \\
\hline$y_{i M}$ & $\begin{array}{l}\text { Límites máximo del ángulo de la articula- } \\
\text { ción } i\end{array}$ \\
\hline$G_{J}$ & Matriz de agarre Jacobiana \\
\hline distance $_{\max }$ & $\begin{array}{l}\text { Distancia máxima del centro de masa del } \\
\text { objeto a cualquier punto en el contorno del } \\
\text { objeto }\end{array}$ \\
\hline Area $a_{\max }$ & $\begin{array}{l}\text { Área máxima posible de la mano calculada } \\
\text { como el área del polígono cuando la mano } \\
\text { está completamente abierta }\end{array}$ \\
\hline Volume $_{\max }$ & $\begin{array}{l}\text { Volumen máximo de la envolvente convexa } \\
\text { de las fuerzas primitivas }\end{array}$ \\
\hline
\end{tabular}

\subsection{OBJETOS}

Para la de medición de las métricas de calidad en los modelos de manos mientras agarran objetos, un subconjunto de 125 objetos ha sido seleccionado de la base de datos de objetos del KIT [23], con el añadido de un modelo de esfera simple. Los objetos incluyen cajas de cereales, botellas de refresco, vasos, juguetes, recipientes de comida, figurillas, comida empaquetada, etc. En la figura 2 se muestra un subconjunto de los objetos seleccio-
Cuadro 3: Especificaciones de los modelos de manos.

\begin{tabular}{|c|c|c|c|}
\hline Mano & Dedos & Articulaciones & Actuadores \\
\hline Shadow & 5 & 17 & 7 \\
\hline Tact & 5 & 11 & 6 \\
\hline Flexy & 5 & 14 & 5 \\
\hline Mich & 5 & 6 & 2 \\
\hline$\overline{\mathrm{PR} 2}$ & 2 & 4 & 2 \\
\hline Barrett & 3 & 7 & 4 \\
\hline Schunk & 3 & 7 & 7 \\
\hline Model T & 4 & 8 & 4 \\
\hline
\end{tabular}

nados

Figura 2: Muestra de los distintos objetos utilizados.

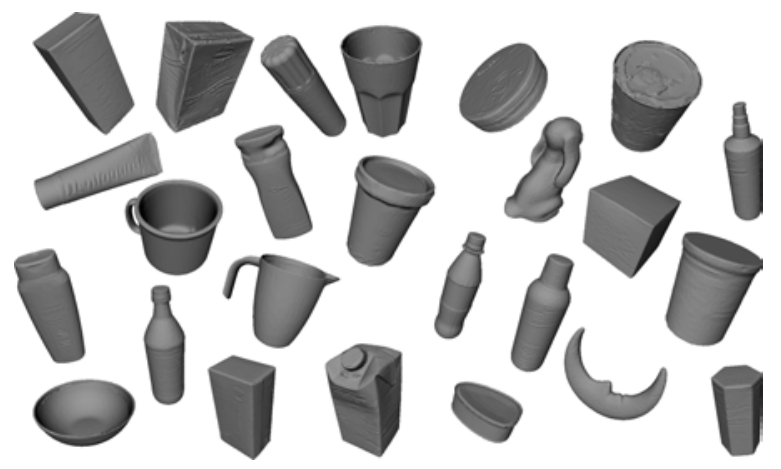

\subsection{HERRAMIENTA DE SIMULACIÓN}

La plataforma de simulación elegida para simular y evaluar los agarres utilizando las métricas de calidad es OpenHand [24], una herramienta de simulación desarrollada por los autores en la cual las métricas de calidad seleccionadas han sido implementadas. Esta basada en OpenRAVE [25], una arquitectura abierta que apunta a una integración 
simple de simulación, visualización, planificación, scripting y control de sistemas robóticos.

\subsection{GENERACIÓN DE AGARRES Y MÉTODO DE EVALAUCIÓN}

Por cada par mano/objeto, una serie de configuraciones de agarre son generadas. Inicialmetne, se realiza una evaluación de cierre de fuerza para filtrar aquellos agarres que no sean estables. A continuación los valores de las métricas de calidad son obtenidos para cada configuración de agarre estable. Para generar los agarres entre objetos y la mano, un matriz cuadrada de puntos es generada alrededor del objeto y proyectada sobre su superficie. Desde estos puntos un conjunto de rayos son proyectados hacia el exterior, definiendo distintas vías de aproximación para el agarre.

Esta generación de agarres depende de diferentes parámetros:

- $\delta$ : Distancia entre los puntos de la matriz cuadrada alrededor del objeto

- $\alpha$ : Ángulos entre el rayo de aproximación y la normal a la superficie del objeto,. Dependiendo de su valor, un conjunto de ratos de aproximación oblicuos es generado.

- $\theta$ : Distancia a lo largo del rayo de aproximación en la que se encuentra el punto de referencia para ubicar la mano

- $N_{r}$ : Ángulos de rotación de la mano sobre el punto de referencia para el agarre.

La table 4 resume los parámetros de configuración titlizados en OpenRAVE para generar agarres. Debido a que el número posible de agarres en cada par mano/objeto puede ser del orden de decenas de miles, un subconjunto aleatorio de hasta 1000 agarres es seleccionado para cada mano y objeto. como consecuencia de la evaluación inicial de cierre de fuerza para obtener tan sólo agarres estables, es posible tener modelos de manos en los cuáles ni siquiera sea posible generar este conjunto de mil agarres aleatorios. Este procedimiento es reptido para cada par mano/objeto, asegurando que los resultados estadísticos son independientes del objeto.

\subsection{EXPERIMENTOS}

El estudio se subdivide en tres experimentos.

\subsubsection{Estudio de Correlación}

Mediante el método de generación de agarres descrito anteriormente, se realiza un estudio de correlación con los resultados de las métricas para
Cuadro 4: Parámetros utilizados para generar la bases de datos de agarres

\begin{tabular}{ll}
\hline Parámetro & Valor \\
\hline$\delta$ espacio delta $(\mathrm{m})$ & 0.01 \\
$\alpha$ ángulo alfa $(\mathrm{rad})$ & $\Pi / 3$ \\
$\theta$ distancia de separación $(\mathrm{m})$ & {$[0.01,0.02]$} \\
$N_{r}$ ángulos de rotación $(\mathrm{rad})$ & {$[0, \Pi / 2, \Pi, 3 \Pi / 2]$} \\
\hline
\end{tabular}

cada grupo de manipuladores artificiales. Dada la cantidad de valores extremos en la evaluación de los agarres, la correlación entre métricas es calculada utilizando el coeficiente de correlación de Spearman. Este estudio permite reducir el número de métricas necesarias para evaluar agarres, pero también indagar entre las diferentes correlaciones entre métricas de acuerdo a los distintos diseños de manos artificiales.

\subsubsection{Estudio de Variabilidad}

Con los mismos datos obtenidos en el estudio de correlación, se realiza el estudio de variabilidad. En este estudio obtenemos valores estadísticos (percentiles, máximos, mínimos y medias) sobre las métricas, de acuerdo a los diferentes modelos de manos artificiales.

Utilizando estos valores podemos definir umbrales sobre los que normalizar las métricas de acuerdo a sus percentiles en distintos conjuntos de manos. Esto permite diferencia entre dos rango de variación de métricas, dependiendo en que tipo d emano artificial estamos utilizando.

\subsubsection{Estudio de Sensibilidad}

Para este estudio tan sólo 20 agarres aleatorios son generados siguiendo las metodologías previamente descritas para cada par mano/objeto. Para cada agarre generado, se realizan 50 variaciones en la posición del manipulador, con el objetivo de simular los errores de posicionamiento y/o percepción cuando se trata de trasladar los agarres del simulador al mundo real. las variaciones en cada posición de agarre son el resultado de traslaciones en el rango de $\pm 1 \mathrm{~cm}$ y rotaciones en el rango de \pm 0.1 radianes a lo largo de los ejes $X, Y, Z$ (fig. $3)$.

Con los datos obtenidos, un Índice de Sensibilidad para cada métrica y mano es calculado como la desviación estándar entre la evaluación de calidad obtenida en el agarre original y las evaluaciones en las posiciones modificadas:

$$
I S=\frac{1}{n} \sum_{x=1}^{n} \sigma_{x}
$$


donde $n$ es el número de agarres y $\sigma_{x}$ la desviación estándar calculada como:

$$
\sigma_{x}=\frac{1}{n_{v}} \sqrt{\sum_{i=1}^{n_{v}}\left(x_{i}-x_{0}\right)^{2}}
$$

donde $n_{v}$ es el número de variaciones, que cumplen la clausura de fuerza, respecto al agarre de referencia, $x_{0}$ el valor de la métrica en el agarre de referencia y $x_{i}$ el valor de la métrica calculado en cada variación.

El $I S$ está expresado en porcentajes $\left(I S_{N}\right)$ utilizando los rangos de las métricas obtenidos en el estudio de variabilidad:

$$
I S_{N}=\frac{I S}{p r c 90-\operatorname{prc10}} 100 \%
$$

donde prc10 y prc90 son los umbrales máximo y mínimo propuestos, obtenidos en cada grupo de modelos de manos.

Finalmente, se calcula un Índice de Sensibilidad Global (ISG) para cada métrica en ambos conjuntos de modelos de manos, este ha sido obtenido al igual que el IS previo, pero teniendo en cuenta las desviaciones estándar para todas las manos en cada grupo:

$$
I S G=\frac{1}{n_{o}} \sum_{i=1}^{n}\left(I S_{i}\right)
$$

donde $n_{o}$ es el numero de manos y $I S_{i}$ el índice de Sensibilidad obtenido en cada una de ellas.

Con los datos obtenidos en este estudio es posible definir un índice de robustez para cada mano, pero también ver que métricas son más robustas en función del tipo de mano artificial utilizado.

Figura 3: Modificaciones en la posición de la mano a lo largo de sus ejes.

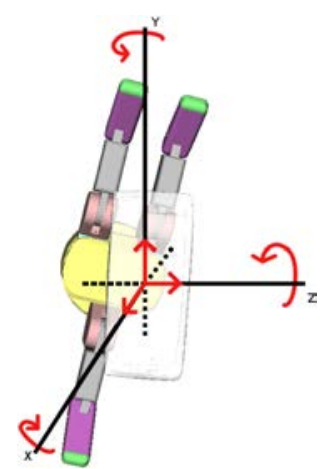

\section{RESULTADOS}

\subsection{ESTUDIO DE CORRELACIÓN}

La tabla 5 muestra la correlación entre los valores de todas las métricas para las pinzas robóticas clásicas, mientras que la tabla 6 muestra esta correlación entre las manos antropomorfas.

De acuerdo a estos datos, en ambos conjuntos de manipuladores, las métricas del grupo A, se pueden agrupar e solo una, en este caso elegimos la métrica $Q_{A 1}$, la cual es una de las que tiene mayores índices de correlación. las métricas del grupo C parecen tener también buena correlación, por lo que las podemos agrupar en $Q_{C 2}$. para el resto de métricas no se observan correlaciones significativas.

Cuadro 5: Correlación de las Métricas de Calidad para las Pinzas Robóticas.

\begin{tabular}{lrrrrrrrrrr}
\hline & $Q_{A 1}$ & $Q_{A 2}$ & $Q_{A 3}$ & $Q_{B 1}$ & $Q_{B 2}$ & $Q_{B 3}$ & $Q_{C 1}$ & $Q_{C 2}$ & $Q_{D 1}$ & $Q_{D 2}$ \\
\hline$Q_{A 1}$ & 1.00 & \multicolumn{1}{c}{} & & & & & & & & \\
$Q_{A 2}$ & 0.93 & 1.00 & & & & & & & & \\
$Q_{A 3}$ & 0.99 & 0.87 & 1.00 & & & & & & & \\
$Q_{B 1}$ & 0.01 & 0.01 & 0.01 & 1.00 & & & & & & \\
$Q_{B 2}$ & 0.30 & 0.47 & 0.23 & -0.09 & 1.00 & & & & & \\
$Q_{B 3}$ & -0.25 & -0.33 & -0.21 & 0.02 & -0.25 & 1.00 & & & & \\
$Q_{C 1}$ & 0.23 & 0.36 & 0.18 & 0.25 & 0.17 & -0.14 & 1.00 & & & \\
$Q_{C 2}$ & 0.46 & 0.52 & 0.42 & 0.21 & 0.21 & -0.22 & 0.72 & 1.00 & & \\
$Q_{D 1}$ & 0.13 & 0.22 & 0.12 & -0.01 & 0.52 & -0.33 & 0.41 & 0.50 & 1.00 & \\
$Q_{D 2}$ & -0.43 & -0.50 & -0.37 & 0.02 & -0.31 & 0.00 & 0.11 & 0.20 & 0.22 & 1.00 \\
\hline
\end{tabular}

Cuadro 6: Correlación de las Métricas de Calidad para las manos Antropomorfas.

\begin{tabular}{lrrrrrrrrrr}
\hline \multicolumn{2}{c}{$Q_{A 1}$} & $Q_{A 2}$ & $Q_{A 3}$ & $Q_{B 1}$ & $Q_{B 2}$ & $Q_{B 3}$ & $Q_{C 1}$ & $Q_{C 2}$ & $Q_{D 1}$ & $Q_{D 2}$ \\
\hline$Q_{A 1}$ & 1.00 & & & & & & & & & \\
$Q_{A 2}$ & 0.95 & 1.00 & & & & & & & & \\
$Q_{A 3}$ & 0.99 & 0.90 & 1.00 & & & & & & & \\
$Q_{B 1}$ & -0.09 & -0.08 & -0.09 & 1.00 & & & & & & \\
$Q_{B 2}$ & 0.19 & 0.31 & 0.14 & 0.09 & 1.00 & & & & & \\
$Q_{B 3}$ & -0.08 & -0.13 & -0.06 & 0.00 & -0.02 & 1.00 & & & & \\
$Q_{C 1}$ & 0.19 & 0.30 & 0.14 & 0.20 & 0.23 & -0.03 & 1.00 & & & \\
$Q_{C 2}$ & 0.40 & 0.51 & 0.35 & 0.19 & 0.40 & -0.06 & 0.64 & 1.00 & & \\
$Q_{D 1}$ & -0.48 & -0.52 & -0.42 & 0.10 & 0.21 & -0.01 & 0.01 & 0.07 & 1.00 & \\
$Q_{D 2}$ & -0.24 & -0.25 & -0.19 & 0.03 & 0.10 & -0.09 & 0.17 & 0.33 & 0.33 & 1.00 \\
\hline
\end{tabular}

\subsection{ESTUDIO DE VARIABILIDAD}

En este estudio, hasta mil agarres han sido generados y evaluados para cada modelo de manipulador. Para cada uno de ellos, se obtienen los valores de las métricas. La tabla 7 muestra los umbrales para normalizar las diferentes métricas. Estos umbrales escogidos coinciden con los percentiles 10 y 90 obtenidos en ambos conjuntos de manos.

la table 8 muestra los valores estadísticos para todas las manos. Los datos seleccionados son los percentiles 10, 50 y 90. Estos limites indican los rangos típicos de variación para una mano con respecto a cada métrica.

La figura 4 muestra gráficamente una compara- 
Cuadro 7: Thresholds for Normalize Metrics in Artificial Hands

\begin{tabular}{lcccccccc}
\hline & & $Q_{A 1}$ & $Q_{B 1}$ & $Q_{B 2}$ & $Q_{B 3}$ & $Q_{C 2}$ & $Q_{D 1}$ & $Q_{D 2}$ \\
\hline \multirow{2}{*}{ Robotic } & $\mathrm{P} 10$ & 0.05 & 0.35 & 0.03 & 0.29 & 0.00 & 0.33 & 0.00 \\
Grippers & $\mathrm{P} 50$ & 0.20 & 0.68 & 0.07 & 0.50 & 0.01 & 0.51 & 0.00 \\
& $\mathrm{P} 90$ & 0.84 & 0.87 & 0.18 & 0.72 & 0.05 & 0.76 & 0.05 \\
\multirow{2}{*}{ Anthropomorphic } & $\mathrm{P} 10$ & 0.04 & 0.26 & 0.03 & 0.27 & 0.00 & 0.26 & 0.00 \\
Hands & $\mathrm{P} 50$ & 0.23 & 0.57 & 0.09 & 0.43 & 0.02 & 0.47 & 0.00 \\
& $\mathrm{P} 90$ & 0.93 & 0.80 & 0.20 & 0.60 & 0.07 & 0.87 & 0.37 \\
\hline
\end{tabular}

Cuadro 8: Evaluación de Variabilidad en los Manipuladores Robóticos

\begin{tabular}{|c|c|c|c|c|c|c|c|c|c|}
\hline & & $Q_{A 1}$ & $Q_{B 1}$ & $Q_{B 2}$ & $Q_{B 3}$ & $Q_{C 2}$ & $Q_{D 1}$ & $Q_{D 2}$ & Agarres \\
\hline \multirow{3}{*}{ Shadow } & $\overline{\mathrm{P} 10}$ & 0.10 & 0.28 & 0.03 & 0.27 & 0.00 & 0.25 & 0.00 & \multirow{3}{*}{126000} \\
\hline & P50 & 0.56 & 0.58 & 0.08 & 0.42 & 0.02 & 0.33 & 0.00 & \\
\hline & P90 & 1.04 & 0.80 & 0.18 & 0.58 & 0.09 & 0.41 & 0.01 & \\
\hline \multirow{3}{*}{ Tact } & P10 & 0.07 & 0.26 & 0.03 & 0.27 & 0.00 & 0.44 & 0.00 & \multirow{3}{*}{125319} \\
\hline & P50 & 0.28 & 0.58 & 0.09 & 0.45 & 0.01 & 0.62 & 0.00 & \\
\hline & P90 & 0.77 & 0.81 & 0.20 & 0.64 & 0.05 & 0.77 & 0.00 & \\
\hline \multirow{3}{*}{ Flexy } & P10 & 0.07 & 0.20 & 0.03 & 0.27 & 0.00 & 0.20 & 0.00 & \multirow{3}{*}{84744} \\
\hline & P50 & 0.49 & 0.50 & 0.09 & 0.42 & 0.01 & 0.31 & 0.00 & \\
\hline & P90 & 1.07 & 0.78 & 0.20 & 0.59 & 0.05 & 0.44 & 0.00 & \\
\hline \multirow{3}{*}{ Mich } & $\mathrm{P} 10$ & 0.03 & 0.31 & 0.03 & 0.12 & 0.00 & 0.70 & 0.11 & \multirow{3}{*}{111270} \\
\hline & P50 & 0.05 & 0.59 & 0.07 & 0.40 & 0.00 & 0.84 & 0.32 & \\
\hline & P90 & 0.07 & 0.82 & 0.14 & 0.55 & 0.01 & 0.95 & 0.64 & \\
\hline \multirow{3}{*}{ PR2 } & $\overline{\mathrm{P} 10}$ & 0.04 & 0.29 & 0.04 & 0.26 & 0.00 & 0.32 & 0.04 & \multirow{3}{*}{113224} \\
\hline & P50 & 0.06 & 0.66 & 0.09 & 0.42 & 0.00 & 0.65 & 0.05 & \\
\hline & P90 & 0.07 & 0.87 & 0.26 & 0.65 & 0.02 & 0.91 & 0.10 & \\
\hline \multirow{3}{*}{ Barrett } & $\mathrm{P} 1$ & 0.0 & 0.45 & $0 .($ & 0.29 & 0.00 & 0.31 & 0.00 & \multirow{3}{*}{126000} \\
\hline & P50 & 0.16 & 0.71 & 0.05 & 0.54 & 0.01 & 0.37 & 0.00 & \\
\hline & P90 & 0.69 & 0.87 & 0.10 & 0.76 & 0.03 & 0.51 & 0.00 & \\
\hline \multirow{3}{*}{ Schunk } & P10 & 0.11 & 0.43 & 0.02 & 0.31 & 0.01 & 0.40 & 0.00 & \multirow{3}{*}{126000} \\
\hline & P50 & 0.46 & 0.71 & 0.06 & 0.47 & 0.02 & 0.54 & 0.00 & \\
\hline & P90 & 0.90 & 0.89 & 0.12 & 0.69 & 0.07 & 0.67 & 0.00 & \\
\hline \multirow{3}{*}{ ModelT } & P 10 & 0.11 & 0.31 & 0.07 & 0.28 & 0.00 & 0.37 & 0.00 & \multirow{3}{*}{125212} \\
\hline & P50 & 0.48 & 0.63 & 0.14 & 0.46 & 0.01 & 0.55 & 0.00 & \\
\hline & P90 & 0.97 & 0.86 & 0.24 & 0.70 & 0.03 & 0.76 & 0.00 & \\
\hline
\end{tabular}

tiva entre las métricas de validad para distintos modelos de manipuladores.

\subsection{Estudio de Sensibilidad}

La table 9 muestra los IS obtenidos para cada modelo de mano, estos IS están normalizados de acuerdo a los percentiles mostrados en la tabla 7 . Los datos en esta table muestran no solo el IS Normalizado, sino también el porcentaje de variaciones de posición de agarre que cumplen con la clausura de fuerza en cada mano.

Cuadro 9: Índice de Sensibilidad (\%) y modificaciones de la posición de agarre válidas (\%) en distintos manipuladores

\begin{tabular}{lrrrrrrrc}
\hline & $Q_{A 1}$ & $Q_{B 1}$ & $Q_{B 2}$ & $Q_{B 3}$ & $Q_{C 2}$ & $Q_{D 1}$ & $Q_{D 2}$ & válidos \\
\hline Shadow & 27.22 & 9.54 & 34.19 & 22.55 & 41.89 & 11.68 & 3.10 & 17.33 \\
Tact & 16.25 & 9.22 & 28.78 & 27.35 & 19.82 & 12.48 & 1.16 & 12.05 \\
Flexy & 26.65 & 7.60 & 32.20 & 23.81 & 19.59 & 15.16 & 1.94 & 11.29 \\
Mich & 32.53 & 8.35 & 0.19 & 0.21 & 0.00 & 33.48 & 321.32 & 3.13 \\
\hline PR2 & 0.14 & 10.98 & 0.52 & 0.76 & 0.23 & 7.14 & 134.69 & 21.10 \\
Barrett & 18.74 & 15.25 & 14.36 & 33.52 & 15.77 & 11.77 & 1.55 & 22.62 \\
Schunk & 25.03 & 13.50 & 19.51 & 36.00 & 30.86 & 17.97 & 1.74 & 39.64 \\
ModelT & 26.82 & 10.50 & 38.18 & 35.12 & 13.74 & 24.55 & 0.00 & 36.30 \\
\hline
\end{tabular}

Figura 4: Percentiles de las métricas de calidad en los manipuladores robóticos.

(a) $Q_{A 1}$
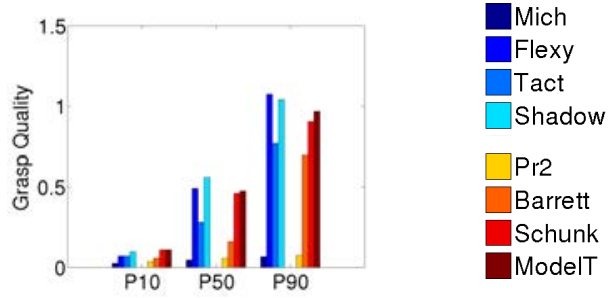

(c) $Q_{B 1}$

(d) $Q_{B 2}$
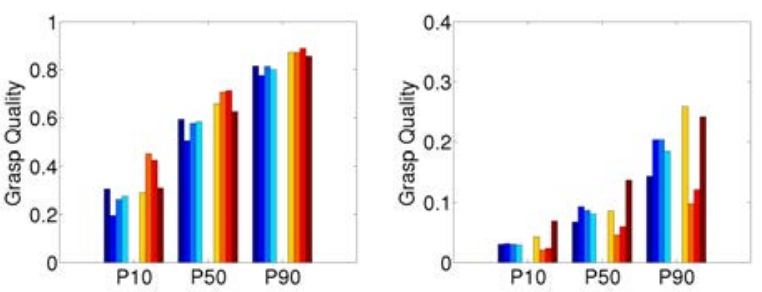

(e) $Q_{B 3}$

(f) $Q_{C 2}$
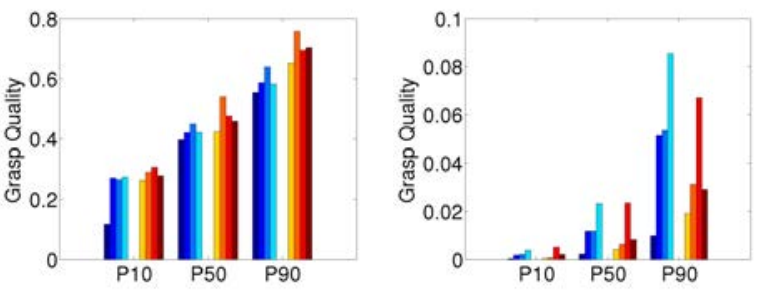

(g) $Q_{D 1}$

(h) $Q_{D 2}$
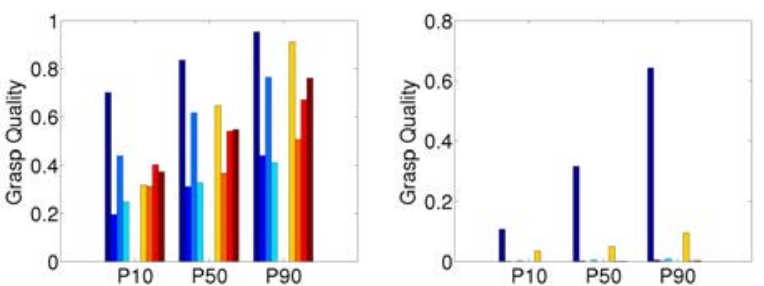

El ISG es mostrado en la tabla 10, diferenciando los manipuladores robóticos de las manos antropomorfas.

\section{DISCUSIÓN}

Las tablas presentadas en el Estudio de Correlación, muestran fuertes correlaciones entre las métricas del Grupo A, y también buenos niveles de correlación para las métricas del Grupo $C$. En esas tablas es también destacable la diferencia de correlación entre las métricas $Q_{C 2}$ y $Q_{D 1}$, dependiendo en el tipo de mano artificial que está siendo evaluada. Como se apuntaba inicialmente por los autores, esto muestra que distintas métricas pueden estar evaluando los mismos aspectos del 
Cuadro 10: Índice de Sensibilidad Global ( \%) para distintos modelos de Manos Artificiales.

\begin{tabular}{cccccccc}
$Q_{A 1}$ & $Q_{B 1}$ & $Q_{B 2}$ & $Q_{B 3}$ & $Q_{C 2}$ & $Q_{D 1}$ & $Q_{D 2}$ & Válidos \\
\hline
\end{tabular}
$\begin{array}{llrllllll}\text { Manos } 25.66 & 8.68 & 23.84 & 18.48 & 20.33 & 18.20 & 81.88 & 10.95\end{array}$

agarre en función del tipo de manipulador que se utiliza.

Los datos del Estudio de Variabilidad, no muestran unas claras diferencias entre el rango de variación de cada métrica, en función del tipo de mano artificial que se este utilizando, teniendo todas las manos rangos de variación similares. tan sólo la métrica $Q_{D 2}$ destaca con altos valores para las manos PR2 y Michelangelo, sin embargo, esto parece estar más relacionado con el hecho de que estas manos tienen tan solo $2 \mathrm{GdL}$, lo que implica que los valores de esta métrica son muy dependientes en el manipulador que este siendo utilizado. Comparando el tipo de manos artificiales, las pinzas robóticas tienen valores ligeramente superiores para las métricas $Q_{B 1}$ y $Q_{B 3}$, lo cuál está directamente relacionado con la distribución de los dedos en dichos manipuladores.

En el caso del Estudio de Sensibilidad, el IS obtenido muestra que la evaluación con las métricas $Q_{A 1}, Q_{B 2}$ y $Q_{C 2}$ es más estable cuando se utilizan pinas robóticas. Por otra parte, las manos antropomorfas son más robustas cuando se evalúan con las métricas $Q_{B 1}$ y $Q_{B 3}$. Este es un resultado esperado, ya que este tipo de métricas tienen en cuenta la distribución geométrica de los puntos de contacto, los cuales varían menos en este tipo de manipuladores artificiales.

Otro punto a destacar del estudio de sensibilidad, es la evaluación de cierre de fuerza en cada modificación de la posición de agarre. Este valor muestra que las manos antropomorfas tienden a fallar mucho más cuando trata de agarrar objetos, ante la presencia de errores en el posicionamiento, lo cuál es realmente difícil de evitar en los sistemas robóticos actuales. Esto sugiere que la distribución opuesta y simétrica de las pinzas robóticas aumenta la probabilidad de que un agarre sea estable, cuando hay incertidumbre en el posicionamiento del manipulador respecto al objeto.

\section{CONCLUSIONES}

En este artículo, un estudio sobre el comportamiento de distintas métricas de acuerdo a distintos modelos de manos artificiales ha sido realizado. Las manos antropomorfas y las pinzas robóticas han sido comparadas de acuerdo a su comportamiento estadístico con diferentes métricas, en re- lación a sus correlaciones, rangos de variación y robustez ante los errores de posicionamiento.

Los resultado obtenidos no son concluyentes. Aunque se observan ciertas diferencias en los valores obtenidos, no se observa una clara tendencia que diferencia las manos antropomorfas de las pinzas robóticas. Parece que el número de GdL sea un parámetro mucho más relevante que la kinemática propia de la mano. De estos datos, resulta evidente que es necesario realizar experimentos mucho más exhaustivos.

Por último, es necesario recordar que este estudio está realizado tan sólo en entornos de simulación. La experimentación con objetos y manos reales es necesaria para proporcionar una referencia sólida a cualquier tipo de resultado.

\section{Agradecimientos}

Esta investigación ha sido parcialmente financiada por Ministerio de Economía y Competitividad (Grant No. DPI2014-60635-R), Ministerio de Educación, Ciencia y Tecnología (Grant No. R31-2008-000-10062-0), Ministerio de Ciencia e Innovación (DPI2011-27846), Generalitat Valenciana (PROMETEO/2009/052, PROMETEOII/2014/028) y Fundació Caixa CastellóBancaixa (P1-1B2011-54 and PI-1B2011-25).

\section{Referencias}

[1] B. Leon, J. Sancho-Bru, N. Jarque-Bou, A. Morales, and M. Roa, "Evaluation of $\mathrm{Hu}-$ man Prehension Using Grasp Quality Measures," International Journal of Advanced Robotic Systems, 2012.

[2] B. Leon, C. Rubert, A. Morales, and Leon, B. and Rubert, C. and Sancho-Bru, J. and Morales, A., "Characterization of Grasp Quality Measures for Evaluating Robotic Hands Prehension," IEEE International Conference on Robotics and Automation, pp. 3688-3693, May 2014.

[3] C. Rubert, B. Leon, and A. Morales, "Grasp Quality Metrics for Robot Hands Benchmarking," Humanoid Robots, 2014 IEEE/RSJ International Conference on, pp. 761-766, Nov. 2014.

[4] Z. Li and S. S. Sastry, "Task-oriented optimal grasping by multifingered robot hands," IEEE Journal of Robotics and Automation,, vol. 4, no. 1, pp. 32-44, Feb. 1987.

[5] B.-H. Kim, S.-R. Oh, B.-J. Yi, and I. H. Suh, "Optimal grasping based on non- 
dimensionalized performance indices," Proceedings of the IEEE International Conference on Intelligent Robots and Systems, vol. 2, pp. 949-956, 2001.

[6] D. Ding, Y.-H. Lee, and S. Wang, "Computation of 3-D form-closure grasps," IEEE Transactions on Robotics and Automation, vol. 17, no. 4, pp. 515-522, Aug. 2001.

[7] J. Ponce, S. Sullivan, A. Sudsang, J.-D. Boissonnat, and J.-P. Merlet, "On Computing Four-Finger Equilibrium and Force-Closure Grasps of Polyhedral Objects," The International Journal of Robotics Research, vol. 16, no. 1, pp. 11-35, 1997.

[8] B. Mirtich and J. Canny, "Easily computable optimum grasps in 2-D and 3-D," Proceedings IEEE International Conference on Robotics and Automation, pp. 739-747, May 1994.

[9] C. Ferrari and J. Canny, "Planning optimal grasps," Proceedings 1992 IEEE International Conference on Robotics and Automation (ICRA), pp. 2290-2295, 1992.

[10] D. G. Kirkpatrick, B. Mishra, and C. K. Yap, "Quantitative Steinitz's theorems with applications to multifingered grasping," Proceedings of the twenty-second annual ACM symposium on Theory of computing, pp. 341-351, 1990.

[11] A. T. Miller and P. K. Allen, "Examples of 3D grasp quality computations," Proceedingsof the IEEE International Conference on Robotics and Automation, vol. 2, no. May, pp. 1240-1246, 1999.

[12] A. Liegeois, "Automatic Supervisory Control of the Configuration and Behavior of Multibody Mechanisms," IEEE Trans. Systems, Man, and Cybernetics, vol. 7, no. 12, pp. 842868, 1977.

[13] J. K. Salisbury and J. J. Craig, "Articulated Hands: Force Control and Kinematic Issues," The International Journal of Robotics Research, vol. 1, no. 1, pp. 4-17, 1982.

[14] J.-O. Kim and P. Khosla, "Dexterity measures for design and control of manipulators," Proceedings IROS Workshop on Intelligent Robots and Systems, no. 91, pp. 758-763, 1991.

[15] Willow Garage, "PR2," http://www.willowgarage.com/pages/pr2.

[16] Barrett Technology Inc., "BarrettHand," http://www.barrett.com/robot/productshand.html.
[17] Schunk GmbH \& Co. KG, "Schunk SDL Hand."

[18] Yale OpenHand Project, "Model T," http://www.eng.yale.edu/grablab/openhand.

[19] P. Slade, A. Akhtar, M. Nguyen, and T. Bretl, "Tact: Design and performance of an open-source, affordable, myoelectric prosthetic hand," 2015 IEEE International Conference on Robotics and Automation (ICRA), pp. 6451-6456, May 2015.

[20] Gyrobot, "Flexy-Hand 2," http://www.gyrobot.co.uk/3d-printing.html.

[21] OttoBock, "Michelangelo Hand," http://www.living-with-michelangelo.com.

[22] Shadow Robot Company, "Shadow Hand," http://www.shadowrobot.com/products/dexteroushand.

[23] Deutsche Forschungsgemeinschaft, "KIT ObjectModels Web Database. Object Models of Household Items," http://i61p109.ira.uka.de/ObjectModelsWebUI/index.php.

[24] B. Leon, A. Morales, and J. Sancho-Bru, From Robot to Human Grasping Simulation, ser. Cognitive Systems Monographs. Springer International Publishing, 2013, vol. 19.

[25] R. Diankov, "Automated Construction of Robotic Manipulation Programs," Ph.D. dissertation, Carnegie Mellon University, Robotics Institute, Aug. 2010. 